\title{
VALORACIÓN SOCIAL DE POLÍTICAS PÚBLICAS DIRIGIDAS A LA NIÑEZ Y ADOLESCENCIA EN LA PROVINCIA DE CÓRDOBA. APLICACIÓN DE ENCUESTAS DE PREFERENCIAS DECLARADAS Y ESTIMACIÓN DE MODELOS DE ELECCIÓN DISCRETA.
}

SOCIAL ASSESSMENT OF PUBLIC POLICIES AIMED AT CHILDREN AND ADOLESCENTS IN THE PROVINCE OF CORDOBA. APPLICATION OF STATED PREFERENCES SURVEYS AND ESTIMATION OF DISCRETE CHOICE MODELS.

\section{JUAN JOSÉ POMPILIO SARTORI ${ }^{2}$ \\ ANA MARÍA ROBLES ${ }^{3}$ \\ JORGE MAURICIO OVIEDO ${ }^{4}$ \\ ARIEL CASTAÑEDA ${ }^{5}$}

RECIBIDO: noviembre 2014 | ACEPTADO: agosto 2015

\section{RESUMEN}

Este estudio presenta estimaciones de modelos de elección discreta utilizando encuestas de preferencias declaradas realizadas a una muestra de trabajadores de la provincia de Córdoba, para luego calcular la valoración social de políticas públicas dirigidas a mejorar el bienestar de niños y adolescentes.

\section{ABSTRACT}

This study presents estimates of discrete choice models using stated preference surveys with a sample of workers in the province of Cordoba, then calculate the social assessment of public policies aimed at improving the welfare of children and adolescents.

\footnotetext{
${ }^{1}$ Profesor Universidad Blas Pascal y Universidad Nacional de Córdoba,jsartori@ubp.edu.ar 
PALABRAS CLAVE: Modelos de elección discreta, preferencias declaradas, deserción escolar, inseguridad alimentaria de niños, indigencia, derechos de niños y adolescentes

KEY WORDS: Discrete choice models, stated preferences, school dropout, food insecurity for children, Indigence, children and adolescents rights.

\section{1-INTRODUCCIÓN}

Existen metodologías que se identifican como tradicionales para la valoración de beneficios y costos de los proyectos o políticas públicas, son aquellas en las cuales los impactos sobre la producción o los propios bienes, implican una identificación plena de sus mercados. Es decir: si el proyecto produce $X$ bienes (o servicios) y utiliza $Y$ factores productivos; estos bienes y factores tienen mercados definidos; y por lo tanto es factible dimensionar el impacto producido con cada mercado y en el bienestar de la sociedad. Sin embargo, a pesar de que las metodologías tradicionales de asignación de precios cuenta de eficiencia (precios sombra) hacen un gran aporte al proceso, existe un vacío generado en la medida que existan externalidades que no puedan ser cuantificadas, y no exista ningún criterio riguroso para establecer si son mayores, menores o iguales a la parte cuantificada. Por ende, no puede establecerse el signo del resultado neto en términos de bienestar de la sociedad.

Tal como plantea Arrow (1986), cuando no existe mercado, hay un vacío de información para la toma de decisiones de los individuos, que ha de completarse con algún tipo de conjetura. El problema es que dichas conjeturas no siempre coindicen con la realidad asociada al fenómeno bajo estudio, y por consiguiente, la toma de decisiones que se deriva de las mismas puede resultar en una asignación no óptima de recursos. Ante este tipo de situaciones, la tarea del evaluador radica en proporcionar instrumentos de análisis que permitan corregir tales desvíos.

Es por lo anterior que se ha vuelto importante el desarrollo de metodologías alternativas para la evaluación económica de proyectos y políticas, que permitan dimensionar la parte no cuantificada, y facilitar el proceso de tomas de decisiones. Entre estas metodologías pueden citarse: análisis costo-eficiencia, ahorro en costos, costos de mitigación, precios hedónicos, costos de viaje (Castro \& Mokate, 2003) y preferencias declaradas, pudiendo clasificar a estas últimas entre técnicas de valoración contingente y estimación de modelos de elección. Los modelos de elección pueden basarse en la respuesta a una elección específica realizada por el entrevistado (modelos de elección discreta propiamente dichos) o en un ordenamiento de preferencias de todas las alternativas incluidas en cada escenario de elección (modelos de elección ordenados).

Según Pere Riera (1994) el método de valoración contingente es una de las técnicas utilizadas para estimar el valor de los bienes (productos o servicios) para los que no existe mercado. El método consiste en simular un mercado mediante encuesta a los consumidores potenciales, preguntando por la máxima cantidad de 
dinero que pagarían por el bien si tuvieran que comprarlo. Esta forma directa de indagación ha sido denominada también "precio de transferencia" (del inglés "transfer price") o "beneficio de transferencia" (del inglés "benefit transfer"). Este método tiene como objetivo que las personas declaren sus preferencias con relación a un determinado bien o servicio, en lugar de realizar estimaciones sobre la base de conductas que se observan en el mercado. En este caso se pregunta directamente sobre la disposición a pagar por un bien o servicio que no posee mercado.

En la encuesta, después de proporcionar la información específica sobre la finalidad del estudio y la situación que se va a valorar, se pregunta al encuestado directamente por su disposición a pagar respecto del bien objeto de análisis. La disposición a pagar estimada es un valor que depende de características observables y no observables del decisor, es decir, desde el punto de vista estadístico es una variable aleatoria.

Este método se aplica generalmente a los servicios ambientales que poseen un valor de existencia significativo.

Por otra parte, los métodos de preferencias declaradas mediante la estimación de modelos de elección discreta también se basan en encuestas presentadas a las personas para que declaren sus preferencias con relación a un determinado bien o servicio. En este caso, se presentan escenarios hipotéticos de elección para que el entrevistado seleccione el que considera de su mayor preferencia.

Estos métodos permiten estimar la máxima disposición a pagar (DAP) por un bien o servicio; o bien la disposición a ser compensado, es decir, disposición a aceptar (DAA)'. La aplicación de estos métodos tiene como objeto la estimación de la función de demanda de un bien que no posee un mercado donde pueda ser transado.

1 En muchos casos se considera inadecuado el uso de la disposición a aceptar debido a que sugiere la posibilidad de que la persona encuestada presente reparos de índole moral; por ejemplo, el hecho de aceptar un pago a cambio del permiso para degradar el medio ambiente. Por otra parte, Carson \& Hanemann (2005) plantea que la elección entre la «disposición a pagar» y la «disposición a aceptar» depende de los derechos de propiedad que existan o se asuman sobre el bien o servicio en cuestión.
Las encuestas de preferencias declaradas y su aplicación con modelos de elección discreta basados en la teoría de la elección del consumidor son un instrumento fundamental para el análisis de la demanda. El punto fundamental que aparta a este paradigma de la teoría económica del consumidor tradicional está relacionado con la idea de que la utilidad se deriva de las propiedades o características de los bienes en vez de hacerlo por los bienes en sí mismos. Este es el llamado "paradigma de la elección" que se encuentra subyacente en el análisis de elección discreta y que une la función de utilidad con los bienes y sus características objetivas (Louviere, Hensher \& Swait, 2000).

El modelo económico teórico postulado para realizar las estimaciones y los pronósticos se basa en la teoría de la utilidad aleatoria, formalizada por Manski (1977). El supuesto básico del modelo de utilidad aleatoria es que un individuo actuando racionalmente puede comparar alternativas y elegir aquella que le reporta el máximo nivel de satisfacción o utilidad, es decir, el individuo elige la alternativa que maximiza su utilidad una vez que se confronta con el ejercicio de elección, dados los atributos de los bienes considerados y sus propias características socio-económicas. El significado "aleatorio" de este modelo se utiliza debido a que en la modelación de las preferencias individuales (utilidades), el analista no posee información completa sobre los argumentos del proceso de decisión, así, una parte de la función de utilidad modelada es mensurable y otra no es directamente mensurable, es aleatoria.

Por lo tanto, el individuo q elegirá la alternativa que le otorgue mayor utilidad que cualquier otra alternativa del conjunto de alternativas disponibles (incluida la alternativa de "no elección"2). Es decir:

$$
V_{j q}=C E A_{j}+\sum_{k} \beta_{k j} x_{k j q}
$$

2 Por lo general se han estimado modelos de elección considerando un conjunto de alternativas determinadas, sin embargo, en los últimos años se ha incluido como una alternativa más que integra el conjunto de elección la denominada "alternativa de no elección" (Dhar, 1997; Haaijer, 1999; Dhar \& Simonson, 2003). 
La parte sistemática (o determinística) de la utilidad individual a menudo se supone como una función lineal aditiva en los atributos en la que los parámetros b se suponen constantes para todos los individuos pero pueden variar entre alternativas. La CEA es la denominada "constante específica de la alternativa" y representa la influencia neta de todas las características no observadas del individuo o de la alternativa en el modelo especificado.

El individuo q elige la alternativa que maximiza su utilidad, es decir:

$$
U_{j q} \geq U_{i q}
$$

donde los subíndices "i" y "j" se refieren a las alternativas de elección disponibles, con $\mathrm{i}=1, \ldots, \mathrm{l} \mathrm{e}^{i \neq j}$.

Esto es,

$$
V_{j q}-V_{i q} \geq \varepsilon_{i q}-\varepsilon_{j q} V_{j q}+\varepsilon_{j q} \geq V_{i q}+\varepsilon_{i q}
$$

Dado que el individuo elige la opción que maximiza su utilidad, se desconoce el valor del segundo miembro de la última desigualdad presentada y el procedimiento para determinar la probabilidad de elegir la alternativa j por el individuo q viene dado por:

$$
P_{j q}=\operatorname{Prob}\left(\varepsilon_{i q}-\varepsilon_{j q} \leq V_{j q}-V_{i q}, \forall i \in i=1, \ldots, I ; i \neq j\right)
$$

Numerosas aplicaciones en el campo del marketing, la economía del transporte y la economía ambiental utilizan la especificación del modelo logit multinomial, que implica que se cumple el supuesto de independencia de las alternativas irrelevantes, la parte no observable de las utilidades se distribuyen de manera idéntica e independiente, no existe correlación serial en el modelo, posee factores de escala constantes para todas las alternativas que se normalizan arbitrariamente igualándolos al valor unitario y los parámetros a estimar son fijos, no aleatorios.

El modelo logit multinomial $\left.\right|^{3}$ puede expresarse como:

3 McFadden (1974), presenta los fundamentos y propiedades econométricas de este modelo denominado originalmente Modelo Logit Condicional.

$$
P_{i}=\frac{e^{\lambda U_{i}}}{\sum_{j=1}^{n} e^{\lambda U_{j}}}
$$

donde:

Ui es la función de utilidad de la alternativa i-ésima, que puede expresarse como: $U_{i}=V_{i}+\varepsilon_{i}$ en la cual $V_{i}$ es la parte determinística de la función de utilidad que usualmente se supone como una función lineal de variables explicativas de la demanda y $\varepsilon_{i}$ es un término de error aleatorio relacionado a los efectos no observables.

$\lambda$ es el factor de escala o parámetro de precisión, que es una función inversa de la desviación estándar de los efectos no observables o errores del modelo $\varepsilon_{i}$. En este modelo, $\lambda$ se supone igual a la unidad.

Asimismo, el modelo logit multinomial permite considerar diferentes conjuntos de elección para cada individuo entrevistado de manera de estimar un modelo como una función de los niveles de servicio de las alternativas y de las características socio-demográficas de los individuos entrevistados.

En este tipo de modelos pueden presentarse algunas limitaciones, como son:

Efectos dinámicos dados por el hecho de que las elecciones en un momento determinado pueden depender de las elecciones realizadas en el pasado, como son por ejemplo los efectos de hábito o aprendizaje. En el caso de trabajar con una secuencia temporal de situaciones de elección, pueden agregarse en la función de utilidad variables exógenas representativas del pasado o el futuro para representar respuestas rezagadas o efectos de hábito y comportamiento anticipado, respectivamente.

Correlación serial existente debido a factores no observables que persisten a través del tiempo o de la secuencia de elecciones, en particular todos los factores relacionados al individuo n, a partir de lo cual 


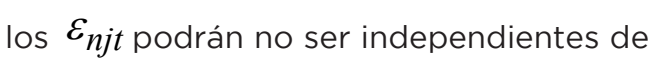
los $\varepsilon_{n j(t-1)}$. Este tipo de correlación resulta de importancia ya que en el caso de trabajar con datos de preferencias declaradas existirán varias respuestas del mismo individuo a diferentes situaciones de elección. La estimación de un modelo logit multinomial mixto de panel permite salvar esta limitación.

\section{2- ELECCIÓN DE PREFERENCIAS DECLARADAS}

A los fines de mejorar la situación de vulnerabilidad de los derechos de los niños resulta importante conocer las preferencias declaradas por la población bajo análisis que permita elaborar un esquema de recaudación de fondos suplementarios a los obtenidos mediante recaudación impositiva tradicional y cumplir con las preferencias de la población.

En este estudio, el diseño del experimento de elección consideró una situación en la que el entrevistado puede elegir entre donar una determinada cantidad de dinero a cuatro diferentes organismos recaudadores (Estado Nacional, Estado Provincial, Estado Municipal, Unicef) o no hacerlo. Se ha utilizado la figura de la donación para evitar utilizar otro mecanismo de financiamiento del gasto, como por ejemplo a través de aumentos de impuestos. Al utilizar la figura de la donación, se supone que el sistema de impuestos no se modifica, por lo tanto, el individuo entrevistado puede elegir donar o no donar sin variación en los tipos de impuestos y alícuotas que enfrenta.

Se trata de un experimento de elección de cinco alternativas, una de las cuales es fija y se corresponde con niveles fijos de los atributos que caracterizan a esa alternativa. Como antes, los niveles fijos utilizados se obtuvieron de la situación actual de los indicadores socioeconómicos de los niños y adolescentes según Tuñón (2011). Los atributos y niveles considerados se exponen a continuación en la Tabla 1. Uno de los atributos se refiere al "aporte o donación mensual" que debería realizarse para obtener mejoras en los demás atributos relacionados a variables relacionadas con la condición socioeconómica de niños y adolescentes en la provincia de Córdoba, a saber:

- $\quad$ Porcentaje de niños entre 5 y 17 años que vive hacinado: con niveles de $12 \%$ y 0\%. (Área: Vivienda).

- $\quad$ Porcentaje de niños entre 5 y 12 años que comparten cama o colchón para dormir: con niveles de 12\%, 6\% y 0\%. (Área: Crianza y socialización).

- Porcentaje de niños menores de 17 años con inseguridad alimentaria (no logra cubrir sus requerimientos nutricionales): con niveles de 10\%. 5\% y 0\%. (Área: Alimentación).

- $\quad$ Porcentaje de niños entre 13 y 17 años que no asiste a nivel secundario: con niveles de 5\% y 0\%. (Área: Educación).

- $\quad$ Porcentaje de niños entre 0 y 17 años en situación de indigencia: con niveles de 6\% y 0\%. (Área: Subsistencia).

- Organismo recaudador: con cuatro niveles, 1. Estado Nacional, 2. Estado Provincial, 3. Estado Municipal y 4. Unicef.

- Aporte o donación mensual: con niveles de $\$ 10, \$ 50, \$ 90$. Cabe señalar, que se restringió el modelo de diseño del experimento de elección, de manera que en cada escenario el nivel de esta variable es el mismo para los diferentes organismos recaudadores y administradores de los fondos donados.

Se diseñó un experimento D-eficiente utilizando el software Ngene (Choicemetrics, 2009), especificando las siguientes funciones de utilidad: 


\begin{tabular}{|c|c|c|c|c|c|}
\hline \multirow{3}{*}{$\begin{array}{l}\text { Atributos de las alternativas } \\
\text { Aporte o donación mensual }\end{array}$} & \multirow{3}{*}{$\begin{array}{l}\text { Nombre de } \\
\text { la variable o } \\
\text { atributo } \\
\text { Ap }\end{array}$} & \multicolumn{4}{|c|}{ Niveles de los atributos de cada alternativa } \\
\hline & & \multirow{2}{*}{$\begin{array}{l}\text { Alternativa } \\
\text { 1. Situación } \\
\text { actual. No } \\
\text { realiza aporte } \\
\text { o donación } \\
\text { específica. } \\
\text { \$0 }\end{array}$} & \multicolumn{3}{|c|}{$\begin{array}{l}\text { Alternativas } 2 \text { a } 5 . \\
\text { Situación en la que } \\
\text { se realiza el aporte o } \\
\text { donación al Estado } \\
\text { Nacional, Estado } \\
\text { Provincial, Estado } \\
\text { Municipal o Unicef. }\end{array}$} \\
\hline & & & $\$ 10$ & $\$ 50$ & $\$ 90$ \\
\hline $\begin{array}{l}\text { Porcentaje de niños entre } 5 \text { y } \\
17 \text { años que vive hacinado }\end{array}$ & Viv & $24 \%$ & $12 \%$ & $0 \%$ & \\
\hline $\begin{array}{l}\text { Porcentaje de niños entre } 5 \text { y } \\
12 \text { años que comparten cama } \\
\text { o colchón para dormir }\end{array}$ & Colch & $18 \%$ & $12 \%$ & $6 \%$ & $0 \%$ \\
\hline $\begin{array}{l}\text { Porcentaje de niños menores } \\
\text { de } 17 \text { años con inseguridad } \\
\text { alimentaria (no logra } \\
\text { cubrir sus requerimientos } \\
\text { nutricionales) }\end{array}$ & Alim & $16 \%$ & $10 \%$ & $5 \%$ & $0 \%$ \\
\hline $\begin{array}{l}\text { Porcentaje de niños entre } 13 \\
\text { y } 17 \text { años que no asiste a nivel } \\
\text { secundario }\end{array}$ & Educ & $10 \%$ & $5 \%$ & O\% & \\
\hline $\begin{array}{l}\text { Porcentaje de niños entre } \\
\text { O y } 17 \text { años en situación de } \\
\text { indigencia }\end{array}$ & Indig & $12 \%$ & $6 \%$ & $0 \%$ & \\
\hline
\end{tabular}

Tabla 1: Atributos y niveles del experimento de elección | Fuente: Elaboración propia.

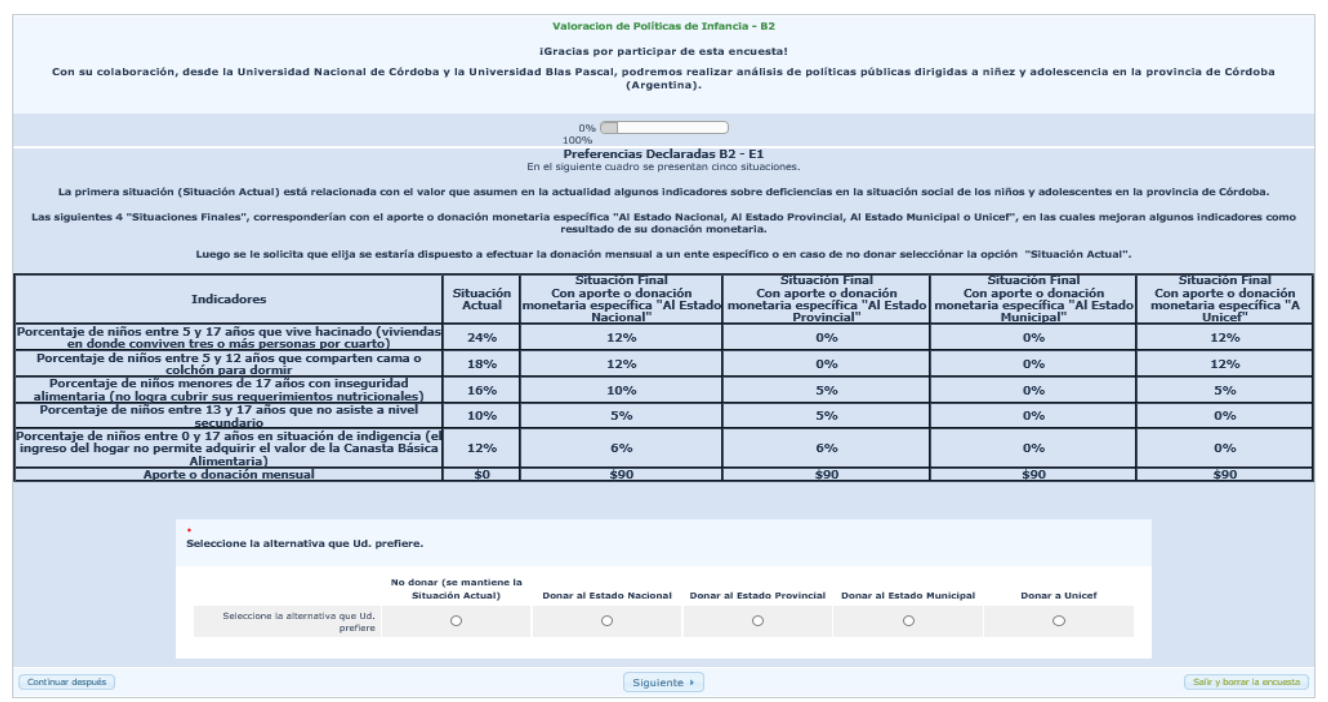

Figura 1: Escenario de elección | Fuente: Elaboración propia. 
$V($ Nacion $)=b 0 N+b 1 . V i v+b 2$. Colch $+b 3$

. Alim $+b 4 . E d u c+b 5$. Indig $+b 6 . A p$

$V($ Provincia $)=b 0 P+b 1$. Viv $+b 2$. Colch + b3. Alim +b4.Educ + b5. Indig + b6.Ap

$V($ Municipio $)=b 0 M+b 1 . V i v+b 2$. Colch + b3. Alim $+b 4 . E d u c+b 5$. Indig $+b 6 . A p$

$V($ Unicef $)=b 0 U+b 1 . V i v+b 2$. Colch $+b 3$

. Alim $+b 4 . E d u c+b 5$. Indig $+b 6 . A p$

$V($ No aporta $)=b 1 . V i v+b 2 . C o l c h+b 3$.

Alim $+b 4 . E d u c+b 5$. Indig $+b 6 . A p$

en las cuales:

$\beta_{\text {ON: }}$ constante específica de la alternativa de elección de donar al Estado Nacional.

$\beta_{o p}$ : constante específica de la alternativa de elección de donar al Estado Provincial.

$\beta_{\text {OM }}$ : constante específica de la alternativa de elección de donar al Estado Municipal.

$\beta_{o u}$ : constante específica de la alternativa de elección de donar a Unicef.

Se generaron diez escenarios de elección en el experimento divididos en dos bloques de cinco escenarios.

En la figura 1 que sigue se presenta a modo de ejemplo un escenario de elección de la encuesta web utilizada.

En el caso de la estimación de modelos de elección discreta, se desea estimar el porcentaje (probabilidad o proporción) de personas que elegirán una alternativa de elección de entre las disponibles.

El marco muestral de esta investigación está conformado por todas las personas mayores de 18 años de edad con ingresos, es decir, que poseen trabajo o ingresos que le permiten decidir si realizarían una donación.

La estrategia de muestreo en una encuesta de preferencias declaradas para estimar modelos de elección discreta debe realizarse cuidadosamente para que la muestra sea representativa de la población bajo estudio.
En la ciudad de Córdoba se realizó un diseño muestral probabilístico por congomerados geográficos, realizado en dos etapas basadas en los hogares, que implicó dividir la ciudad en 74 zonas geográficas que se corresponden con las fracciones poblacionales del Censo de Población del año 2001, seleccionar aleatoriamente un radio censal de cada una de las fracciones y luego una manzana dentro del radio seleccionado. La selección de hogares a encuestar en cada manzana elegida se realizó por medio de muestreo sistemático, hasta completar al menos una encuesta a un trabajador por cada manzana seleccionada. En las ciudades del interior de la provincia que se presentan en la Tabla 2 , se realizó un muestreo no probabilístico del tipo bola de nieve a partir de algunos trabajadores seleccionados.

La muestra a partir con la que se efectuaron las estimaciones quedó conformada por 540 casos (o respuestas de escenarios de elección) correspondientes a 108 encuestados que respondieron la encuesta en el primer semestre de 2014. Con este tamaño de la muestra y considerando muestreo aleatorio simple para todas las ciudades consideradas en el estudio, el error de muestreo ex - post ascendería a $3,5 \%$, con un $90 \%$ de nivel de confianza. Dada la característica no probabilística de la muestra en las ciudades del interior de Córdoba unido a que en algunas de ellas consiguieron realizarse muy pocas encuestas, debe considerarse a este estudio como exploratorio y a la encuesta realizada como encuesta piloto.

En la siguiente tabla se presenta la distribución de casos (o respuestas a escenarios de elección) según ciudad.

Las encuestas se recopilaron vía web en todos los casos. En el caso de la ciudad de Córdoba se realizaron visitas personales a los hogares que permitieron establecer el primer contacto con el hogar seleccionado y tomar las direcciones de email de los trabajadores del hogar, que luego permitieron enviar las invitaciones a participar de la encuesta web realizada en la plataforma LimeSurvey disponible en la Universidad Nacional de Córdoba. En el caso de las ciudades del interior, se tomó contacto con estudiantes universitarios y personal docente de la Universidad $\mathrm{Na}$ - 
cional de Córdoba, de la Universidad Tecnológica Nacional (Facultad Regional Villa María) y de la Universidad Blas Pascal que brindaron información sobre contactos de las diferentes ciudades del interior, a partir de los cuales se aplicó la estrategia de bola de nieve mencionada anteriormente.

\begin{tabular}{|l|c|c|c|}
\hline CIUDAD & FRECUENCIA & PORCENTAJE & $\begin{array}{c}\text { PORCENTAJE } \\
\text { ACUMULADO }\end{array}$ \\
\hline Villa Carlos Paz & 10 & 1,85 & 1,85 \\
Colonia Caroya & 5 & 0,93 & 2,78 \\
Córdoba capital & 415 & 76,85 & 79,63 \\
Mendiolaza & 5 & 0,93 & 80,56 \\
Río Ceballos & 25 & 4,63 & 85,19 \\
Río Cuarto & 5 & 0,93 & 86,11 \\
San Francisco & 10 & 1,85 & 87,96 \\
Unquillo & 5 & 0,93 & 88,89 \\
Villa Allende & 30 & 5,56 & 94,44 \\
Villa María & 25 & 4,63 & 99,07 \\
Villa Nueva & 5 & 0,93 & 100,00 \\
\hline Total & 540 & 100,0 & \\
\hline
\end{tabular}

Tabla 2: Distribución de respuestas según ciudad | Fuente: Elaboración propia.

3- ESTIMACIÓN DE LA

VALORACIÓN SOCIAL DE POLÍTICAS DIRIGIDAS A NIÑEZ Y ADOLESCENCIA EN LA PROVINCIA DE CÓRDOBA. RESULTADOS Y DISCUSIÓN

A partir de las encuestas relevadas, se estimó el modelo especificado logit multinomial en el diseño del experimento de elección.

Las funciones de utilidad del modelo estimado son las siguientes: 
$V($ Nacion $)=b 0 N+b 1 . V i v+b 2$. Colch $+b 3$. Alim $+b 4 . E d u c+b 5$. Indig $+b 6 . A p$

V $($ Provincia $)=b 0 P+b 1$. Viv $+b 2$. Colch $+b 3$. Alim $+b 4 . E d u c+b 5$. Indig $+b 6 . A p$

$V($ Municipio $)=b 0 M+b 1 . V i v+b 2$. Colch $+b 3$. Alim $+b 4 . E d u c+b 5$. Indig $+b 6 . A p$

$V($ Unicef $)=b 0 U+b 1$. Viv $+b 2$. Colch $+b 3$. Alim $+b 4 . E d u c+b 5$. Indig $+b 6 . A p$

$V($ No aporta $)=b 1$. Viv $+b 2$. Colch $+b 3$. Alim $+b 4 . E d u c+b 5$. Indig $+b 6 . A p$

Al estimar el modelo se obtuvieron los resultados que se presentan en la siguiente tabla.

\begin{tabular}{|c|c|c|c|c|c|c|}
\hline \multirow[b]{2}{*}{ Parámetro } & \multicolumn{3}{|c|}{ Logit multinomial } & \multicolumn{3}{|c|}{$\begin{array}{l}\text { Logit multinomial mixto con efectos de } \\
\text { panel para respuestas repetidas }\end{array}$} \\
\hline & $\begin{array}{l}\text { Valor del } \\
\text { parámetro }\end{array}$ & $\begin{array}{l}\text { Estadístico } \\
\text { t robusto }\end{array}$ & $\mathrm{p}$-valor & $\begin{array}{l}\text { Valor del } \\
\text { parámetro }\end{array}$ & $\begin{array}{l}\text { Estadístico t } \\
\text { robusto }\end{array}$ & p-valor \\
\hline$\beta_{O N}$ & $-2,85$ & $-6,53$ & 0,00 & 6,39 & 1,80 & 0,07 \\
\hline$\beta_{O P}$ & $-3,23$ & $-7,38$ & 0,00 & 6,01 & 1,68 & 0,09 \\
\hline$\beta_{O M}$ & $-2,80$ & $-6,14$ & 0,00 & 6,44 & 1,80 & 0,07 \\
\hline$\beta_{o u}$ & $-1,76$ & $-4,13$ & 0,00 & 7,50 & 2,10 & 0,04 \\
\hline$\beta_{1}$ & $-0,0443$ & $-4,67$ & 0,00 & $-0,0452$ & $-4,29$ & 0,00 \\
\hline$\beta_{2}$ & $-0,0467$ & $-4,13$ & 0,00 & $-0,0478$ & $-4,38$ & 0,00 \\
\hline$\beta_{3}$ & $-0,079$ & $-6,12$ & 0,00 & $-0,0799$ & $-5,68$ & 0,00 \\
\hline$\beta_{4}$ & $-0,0239$ & $-1,08$ & 0,28 & $-0,0218$ & $-1,16$ & 0,25 \\
\hline$\beta_{5}$ & $-0,0664$ & $-3,88$ & 0,00 & $-0,0677$ & $-4,23$ & 0,00 \\
\hline$\beta_{6}$ & $-0,00284$ & $-0,68$ & 0,50 & $-0,00394$ & $-0,34$ & 0,73 \\
\hline$\sigma_{\text {panel }}$ & --- & --- & --- & 9,21 & 3,51 & 0,00 \\
\hline \multicolumn{2}{|c|}{ Número de observaciones: } & \multicolumn{2}{|l|}{540} & \multicolumn{3}{|c|}{540} \\
\hline \multicolumn{2}{|c|}{ Rho cuadrado: } & \multicolumn{2}{|l|}{0,169} & \multicolumn{3}{|c|}{0,293} \\
\hline \multicolumn{2}{|c|}{ Rho cuadrado ajustado: } & \multicolumn{2}{|l|}{0,158} & \multicolumn{3}{|c|}{0,280} \\
\hline \multicolumn{2}{|c|}{$\begin{array}{l}\text { Log de verosimilitud } \\
\text { inicial: }\end{array}$} & $-869,096$ & & \multicolumn{3}{|c|}{$-860,096$} \\
\hline \multicolumn{2}{|c|}{ Log de verosimilitud final: } & $-721,814$ & & \multicolumn{3}{|c|}{$-614,554$} \\
\hline \multicolumn{2}{|c|}{$\begin{array}{l}\text { Test de Razón de } \\
\text { verosimilitud: }\end{array}$} & 294,564 & & \multicolumn{3}{|c|}{509,085} \\
\hline
\end{tabular}

Tabla 3: Logit multinomial de elección de donación o aporte para mejorar las condiciones socioeconómicas de niños y adolescentes de la Provincia de Córdoba | Fuente: Elaboración propia basada en resultados de BIOGEME (Bierlaire, 2003 y 2009), utilizando modelos de elección discreta con simulación (Train, 2009). 
Como puede apreciarse se estimó el modelo logit multinomial para la elección de no donar o realizar una donación a diferentes entes recaudadores y el modelo logit multinomial con efectos de panel. Dado que el modelo con efectos de panel para respuestas repetidas resultó válido a juzgar por la significatividad del parámetro estimado spanel, y además arrojó una mejor bondad de ajuste general evidenciada por el coeficiente Rho cuadrado ajustado, este es el mejor modelo que representa las preferencias de la muestra utilizada.

En los modelos logit multinomiales estimados los parámetros resultaron con el signo esperado y en su mayoría significativos considerando un $5 \%$ de nivel de significación. No resultaron significativos (al $5 \%$ de nivel de significación) los coeficientes asociados a las variables "aporte" y "educación". Sin embargo, a los fines de obtener una primera estimación de la valoración subjetiva de las políticas dirigidas a mejorar la calidad de vida de niños y adolescentes en la provincia de Córdoba resulta necesario contar con el parámetro asociado a la variable "aporte" que resultó con el signo negativo esperado, razón por la cual se ha considerado como válido el modelo estimado, a los fines de mostrar los primeros resultados obtenidos con esta metodología en la provincia de Córdoba y posiblemente a nivel mundial. Sin lugar a dudas, resulta necesario ampliar el número de casos de la muestra a los fines de mejorar la eficiencia de los parámetros estimados, tarea que podrá realizarse en próximas investigaciones.

A partir de estas estimaciones, puede calcularse la valoración monetaria subjetiva de cada uno de los atributos considerados en la elección de realizar un aporte o contribución.

Como es sabido, una manera de establecer la importancia relativa de los atributos en una estimación de demanda de elección discreta es calcular la valoración de un atributo en términos de otro atributo utilizado como numerario, tal como el precio o costo de la alternativa. Así, por ejemplo, para obtener la valoración subjetiva de una reducción de un $1 \%$ en el porcentaje de niños que vive hacinado, se divide el valor estimado del parámetro relacionado a la variable Viv ( $\beta 1$ ) por el valor estimado del parámetro asociado a la variable que representa el aporte o donación mensual ( $\beta 6)$.

Las valoraciones subjetivas calculadas para los distintos indicadores considerados a partir de los modelos estimados son las que se exponen en la siguiente tabla.

Según los resultados de ambos modelos estimados, existe una mayor valoración de las políticas dirigidas a disminuir la inseguridad alimentaria y la indigencia, seguidas por las valoraciones de las políticas dirigidas a reducir el hacinamiento (tanto el hacinamiento propiamente dicho como la situación de compartir cama o colchón para dormir) y luego la deserción escolar en el nivel de enseñanza secundario.

Asimismo, debe señalarse que existe una mayor preferencia por efectuar donaciones a Unicef en relación a los demás organismos estatales recaudadores y administradores de los fondos que los encuestados estarían dispuestos a donar. 


\begin{tabular}{|l|c|c|}
\hline Modelo estimado: & $\begin{array}{l}\text { Logit } \\
\text { multinomial }\end{array}$ & $\begin{array}{l}\text { Logit multinomial } \\
\text { mixto con efectos de } \\
\text { panel para respuestas } \\
\text { repetidas }\end{array}$ \\
\hline $\begin{array}{l}\text { Valoración subjetiva de una reducción de un 1\% en } \\
\text { el porcentaje de niños que vive hacinado: }\end{array}$ & $\$ 15,60$ & $\$ 11,47$ \\
\hline $\begin{array}{l}\text { Valoración subjetiva de una reducción de un 1\% } \\
\text { en el porcentaje de niños que comparten cama o } \\
\text { colchón para dormir: }\end{array}$ & $\$ 16,44$ & $\$ 12,13$ \\
\hline $\begin{array}{l}\text { Valoración subjetiva de una reducción de un 1\% en } \\
\text { el porcentaje de niños con inseguridad alimentaria: }\end{array}$ & $\$ 27,28$ & $\$ 20,28$ \\
\hline $\begin{array}{l}\text { Valoración subjetiva de una reducción de un 1\% } \\
\text { en el porcentaje de niños que no asiste a nivel } \\
\text { secundario: }\end{array}$ & $\$ 8,42$ & $\$ 5,53$ \\
\hline $\begin{array}{l}\text { Valoración subjetiva de una reducción de un 1\% en } \\
\text { el porcentaje de niños en situación de indigencia: }\end{array}$ & $\$ 23,38$ & $\$ 17,18$ \\
\hline
\end{tabular}

Tabla 4: Valoración Social de Políticas dirigidas a niñez y adolescencia en la Provincia de Córdoba | Fuente: Elaboración propia.

\section{4-CONCLUSIONES}

Con el presente trabajo se pudo observar la importancia de la valoración social subjetiva de las políticas públicas por parte de los miembros de una comunidad.

En diferentes artículos se ha analizado la evolución del gasto público dirigido a niñez en la Provincia de Córdoba (Blanco y Zen, 2005; Sartori, Oviedo y Robles, 2013). La evolución creciente del gasto público dirigido a niñez y adolescencia en términos del producto bruto geográfico de la provincia de Córdoba o el gasto por niño en valores monetarios constantes, podría suponer la existencia o la generación de una percepción favorable por parte de la población hacia las políticas aplicadas por el Estado.
A partir del presente estudio de preferencias declaradas, se relacionó la disposición a realizar una donación monetaria mensual que resultara en una mejora de las condiciones de vida de niños y adolescentes en la provincia de Córdoba. Dicho trabajo fue efectuado a través de una encuesta a adultos y estimando a partir de la misma la valoración subjetiva y disposición a realizar aportes (donaciones) para implementar políticas específicas que permitan mejorar las condiciones socioeconómicas de los niños y adolescentes de Córdoba. Como conclusión se observa que las preferencias de la población en relación a políticas específicas dirigidas a mejorar la situación socioeconómica de niños y adolescentes podrían ser diferen- 
tes a la valoración subjetiva de quienes implementan las políticas. Es probable que la evaluación realizada de la población hacia el Estado posiblemente considere indicadores diferentes a los que puedan surgir del análisis de los resultados de una Ejecución Presupuestaria o sobre la cuenta Ahorro - Inversión - Financiamiento del gobierno. Ello surge a partir de los resultados obtenidos que muestran que existe una mayor preferencia por efectuar donaciones a Unicef en relación a los demás organismos estatales recaudadores y administradores de los fondos donados. Asimismo, es de destacar que existe por parte de la población una mayor valoración de las políticas dirigidas a disminuir la indigencia y la inseguridad alimentaria, seguidas por las valoraciones de las políticas dirigidas a reducir el hacinamiento (tanto el hacinamiento propiamente dicho como la situación de compartir cama o colchón para dormir) y la deserción escolar.

Para ello sería importante avanzar por parte del Estado en el registro anual de la situación de los indicadores relacionadas a las condiciones socioeconómicas de niños y adolescentes para luego calcular de qué manera los aumentos en el gasto público se vinculan con mejoras en los valores de los indicadores relevados. Luego, podrán compararse los incrementos específicos de gasto con la valoración subjetiva social que le otorga la comunidad a ese gasto. De esta manera, se podrían medir los beneficios sociales resultantes en el marco analítico de la evaluación económica y social de políticas de gobierno, lo que permitiría tomar decisiones de asignación del gasto público a aquellas políticas que arrojen mayores beneficios sociales, contribuyendo así al desarrollo económico. 
ARROW K., "Rationality of self and others in an economic system", Journal of Business, Cambridge, Estados Unidos, 1986.

BIERLAIRE M., "Biogeme: a free package for the estimation of discrete choice models, proceedings of the 3rd swiss transportation research conference", Nueva York, Estados Unidos, 2003.

BIERLAIRE M., "Estimation of discrete choice models with BIOGEME 1.8", http://biogeme.epfl.ch/v18/tutorialv18.pdf, 2009.

BLANCO F. \& Zen S., "Gasto público dirigido a la niñez y adolescencia en la provincia de Córdoba, 2000-2004: un estudio sobre la inversión de recursos públicos provinciales", Fundación Arcor y UNICEF, Córdona, Argentina, 2005.

CARSON R. \& HANEMANN W. "Contingent valuation: handbook of environmental economics, 2", California, Estados Unidos, 2005.

CASTRO R. \& MOKATE K., "Evaluación económica y social de proyectos de inversión", Alfaomega, Nueva York, Estados Unidos, 2003.

CHOICECMETRICS, "NGene 1.0 user manual \& reference guide, the cutting edge in experimental design", Nueva York, Estados Unidos, 2009.

DHAR R. \& SIMONSON I., "The effect of forced choice on choice", journal of marketing research, Chicago, Estados Unidos, 2003.

DHAR R., "Consumer preference for a no-choice option", journal of consumer research, Oxford, Inglaterra, 1997.

HAAIJER M., "Modeling conjoint choice experiments with the probit model", The Netherlands, Labyrint Publications, Nueva York, Estados Unidos, 1999.
LOUVIERE J., HENSHER D. \& SWAIT J., "Stated choice methods. Analysis and application", Cambridge University Press, Cambridge, Estados Unidos, 2000.

MANSKI C., "The structure of random utility models, theory and decision”, Nueva York, Estados Unidos, 1977.

MCFADDEN D., "Conditional logit analysis of qualitative choice behaviour, frontiers of econometrics", Academic Press, Zarembka, Nueva York, Estados Unidos, 1974.

PERE RIERA M., "Manual de valoración contingente, Ministerio de Economía y Hacienda", Madrid, España, 1994.

SARTORI, J., OVIEDO J. \& ROBLES A., "XLVIII reunión anual de la Asociación Argentina de Economía Política, una estimación de la valoración social de las políticas públicas dirigidas a niñez y adolescencia en la provincia de Córdoba aplicando encuestas de preferencias declaradas", Universidad Nacional de Rosario y Consejo Profesional de Ciencias Económicas de la Provincia de Santa Fe, Rosario, Argentina, 2013.

TRAIN, K., "Discrete choice methods with simulation", Cambridge University Press, http://eml.berkeley.edu/ books/choice2.html, 2009.

TUÑÓN I., "Situación de la infancia a inicios del bicentenario: un enfoque multidimensional y de derechos, barómetro de la deuda social de la infancia", Universidad Católica Argentina, http://bibliotecadigital. uca.edu.ar/repositorio/investigacion/situacion-infancia-inicios-bicentenario-multidimensional.pdf, Buenos Aires, Argentina, 2011. 